Acta Crystallographica Section E

Structure Reports

Online

ISSN 1600-5368

\section{Hazel S. Wilkinson and William T. A. Harrison*}

Department of Chemistry, University of Aberdeen, Meston Walk, Aberdeen AB24 3UE, Scotland

Correspondence e-mail:

w.harrison@abdn.ac.uk

\section{Key indicators}

Single-crystal X-ray study

$T=293 \mathrm{~K}$

Mean $\sigma(\mathrm{C}-\mathrm{C})=0.003 \AA$

$R$ factor $=0.027$

$w R$ factor $=0.069$

Data-to-parameter ratio $=26.9$

For details of how these key indicators were automatically derived from the article, see http://journals.iucr.org/e.
(C) 2005 International Union of Crystallography Printed in Great Britain - all rights reserved

\title{
Creatininium dihydrogenarsenate
}

The title compound, $\left(\mathrm{C}_{4} \mathrm{H}_{8} \mathrm{~N}_{3} \mathrm{O}\right)\left[\mathrm{H}_{2} \mathrm{AsO}_{4}\right]$, contains a network of creatininium cations and dihydrogenarsenate anions [mean As $-\mathrm{O}=1.681$ (2) $\AA$ ]. The crystal packing involves anion-toanion $\mathrm{O}-\mathrm{H} \cdots \mathrm{O}$, cation-to-anion $\mathrm{N}-\mathrm{H} \cdots \mathrm{O}$ and cation-tocation $\mathrm{N}-\mathrm{H} \cdots \mathrm{O}$ hydrogen bonds, resulting in a chain structure.

\section{Comment}

The title compound, (I) (Fig. 1), was prepared as part of our ongoing structural studies of hydrogen-bonding interactions in protonated-amine (di)hydrogen arsenate molecular salts (Wilkinson \& Harrison, 2004; Todd \& Harrison, 2005). The $\left[\mathrm{H}_{2} \mathrm{AsO}_{4}\right]^{-}$dihydrogenarsenate group in (I) shows a normal tetrahedral geometry [mean As-O = 1.681 (2) $\AA$ ] , with the protonated As1-O3 and As1-O4 vertices showing their usual lengthening relative to the unprotonated As1-O1 and As1-O2 bonds, which have formal partial double-bond character (Table 1).<smiles>CN1CC(=O)NC1=[NH2+]</smiles>

(I)

The creatininium cation is approximately planar [r.m.s. deviation for the non- $\mathrm{H}$ atoms $=0.031 \AA$; maximum deviation from the mean plane $=0.0597$ (16) $\AA$ for N2]. The three $\mathrm{C} 1-$ $\mathrm{N}$ bond distances (Table 1 ) are distinctly different, with $\mathrm{C} 1-$ N1 much longer than the other two. This configuration perhaps indicates that the canonical form of the molecule, with a formal double bond in the $\mathrm{C} 1-\mathrm{N} 1$ position and a formal positive charge on $\mathrm{N} 1$, is of less importance than the forms that place the double bond in the $\mathrm{C} 1-\mathrm{N} 2$ and $\mathrm{C} 1-\mathrm{N} 3$ positions and the positive charge on the respective $\mathrm{N}$ atoms. However, the rather short $\mathrm{C} 2-\mathrm{N} 1$ bond length suggests that some conjugation involving the $\mathrm{C} 2=\mathrm{O} 5$ group may also be significant. In the structure of creatininium dipicolinate monohydrate (Moghimi et al., 2004), the creatininium cation is constrained to be planar by mirror symmetry and an almost identical pattern of $\mathrm{C}-\mathrm{N}$ bond lengths is observed.

As well as electrostatic attractions, the component species in (I) interact by means of a network of cation-to-anion $\mathrm{N}-$ $\mathrm{H} \cdots \mathrm{O}$, anion-to-anion $\mathrm{O}-\mathrm{H} \cdots \mathrm{O}$ and cation-to-cation $\mathrm{N}-$ $\mathrm{H} \cdots \mathrm{O}$ hydrogen bonds (Table 2). The $\left[\mathrm{H}_{2} \mathrm{AsO}_{4}\right]^{-}$units are linked into polymeric chains (Fig. 2) propagating along [100] by way of inversion-generated pairs of $\mathrm{O} 3-\mathrm{H} 1 \cdots \mathrm{O} 2^{\mathrm{i}}$ and
Received 17 May 2005 Accepted 20 May 2005 Online 28 May 2005 


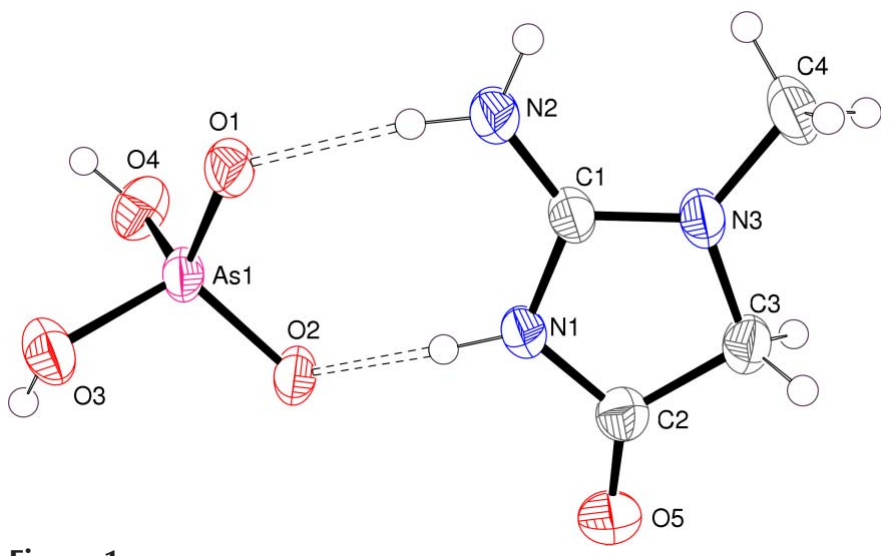

Figure 1

View of the asymmetric unit of (I), showing $50 \%$ probability displacement ellipsoids, with hydrogen bonds indicated by dashed lines.

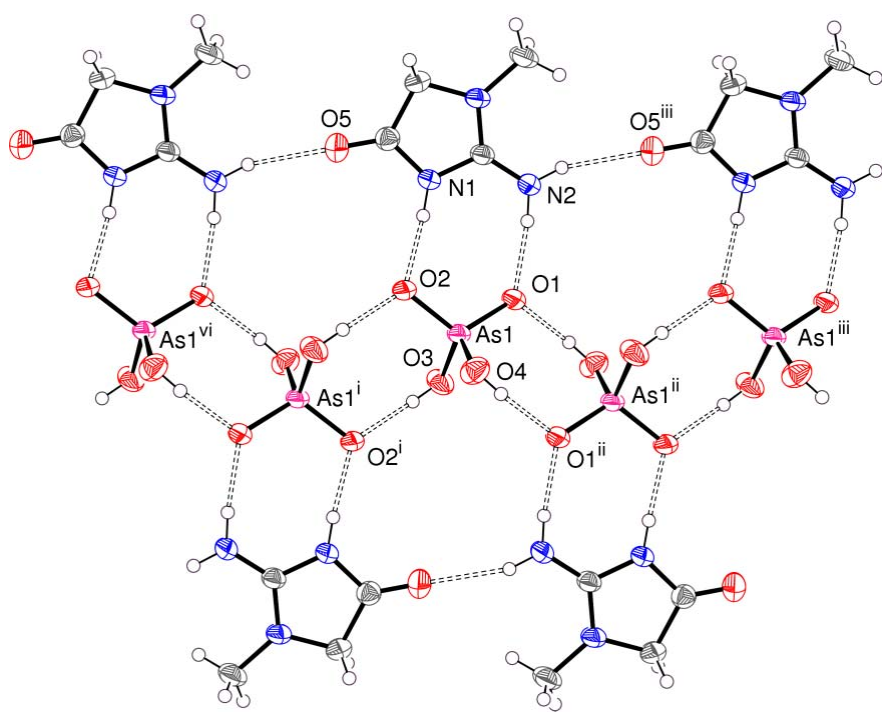

Figure 2

Detail of a hydrogen-bonded chain in (I). Hydrogen bonds are indicated by dashed lines. [Symmetry codes as in Table 2; additionally, (vi) $x-1, y$, $z$.

$\mathrm{O} 4-\mathrm{H} 2 \cdots \mathrm{O} 1^{\mathrm{ii}}$ bonds (see Table 2 for symmetry codes). In graph-set notation (Bernstein et al., 1995), each inter-tetrahedral linking motif corresponds to an $R_{2}^{2}(8)$ loop. The As1 $\cdots A s 1^{\text {i }}$ and As1 $\cdots$ As $1^{\text {ii }}$ separations are $4.0608(3)$ and 3.9286 (3) ̊̊, respectively.

The organic species interacts with the dihydrogenarsenate chains by way of two $\mathrm{N}-\mathrm{H}$. . O hydrogen bonds (Fig. 1), such that both sides of each [100] chain are decorated by the creatininium cations. The third creatininium $\mathrm{N}-\mathrm{H}$ group is involved in a cation-to-cation $\mathrm{N}-\mathrm{H}$...O bond (Fig. 2) that appears to reinforce the chains. Overall, a chain structure along the $a$ axis arises for (I), as shown in Fig. 3. Atoms O1 and $\mathrm{O} 2$ accept two hydrogen bonds each (bond angle sums about these atoms are 358.3 and $359.3^{\circ}$, respectively). A PLATON (Spek, 2003) analysis of (I) flagged the possible presence of two short $\mathrm{C}-\mathrm{H} \cdots \mathrm{O}$ interactions (Table 2), although their structural significance is not clear.

PLATON also flagged a short $\mathrm{C} 2 \cdots \mathrm{C} 2{ }^{\mathrm{v}}$ [symmetry code: (v) $-x, 1-y,-z]$ intermolecular contact of $3.158(3) \AA$,

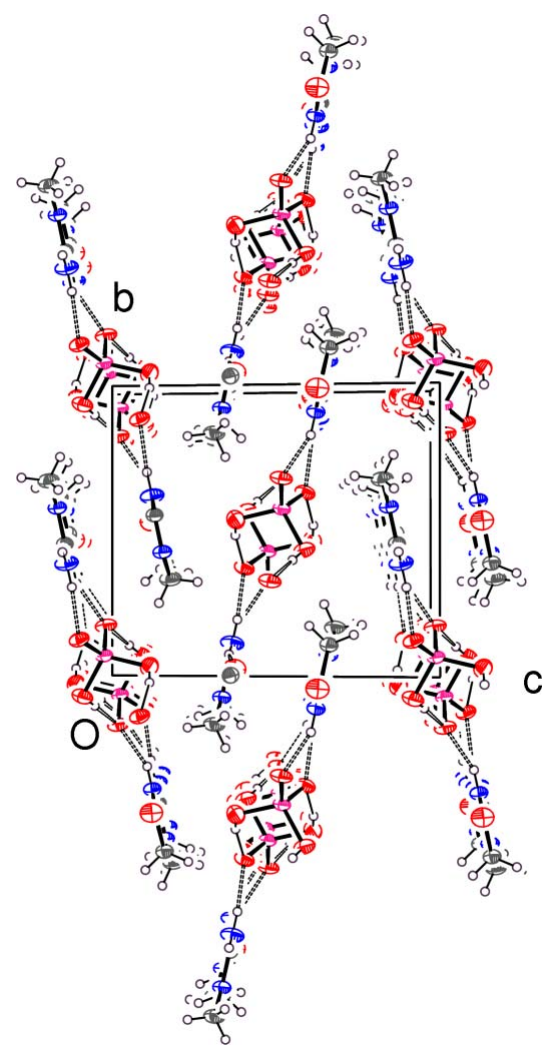

Figure 3

Projection of the packing of (I) along the $a$ axis. Hydrogen bonds are indicated by dashed lines.

compared with a van der Waals radius sum of $3.40 \AA$ (Bondi, 1964). This close contact might arise as part of a carbonylcarbonyl interaction, as described by Allen et al. (1998). In the notation of these workers, the situation in (I) corresponds to a 'sheared antiparallel' or motif II interaction (Fig. 4), generated by a centre of symmetry. Here, the $\mathrm{O} 5=\mathrm{C} 2 \cdots \mathrm{O}^{\mathrm{v}}$ and $\mathrm{C} 2=\mathrm{O} 5 \cdots \mathrm{C}^{\mathrm{v}}$ interaction angles are $100.50(14)$ and $79.50(13)^{\circ}$, respectively, compared with the nominal values of $2 \times 90^{\circ}$ for a perfect rectangle of the four constituent atoms. This is slightly more distorted than the mean $\mathrm{O}=\mathrm{C} \cdots \mathrm{O}$ and $\mathrm{C}=\mathrm{O} \cdots \mathrm{C}$ angles of $96.5(4)$ and $83.5(4)^{\circ}$ based on 553 contributors, as cited by Allen et al. (1998). The $\mathrm{C} 2 \cdots \mathrm{O}^{\mathrm{v}}$ separation of 3.147 (3) $\AA$ in (I) is slightly less than the C... van der Waals separation of $3.22 \AA$.

The dihydrogenarsenate chain motif in (I) replicates that seen in $t$-butylammonium dihydrogenarsenate (Wilkinson \& Harrison, 2004). However, a different cation-to-anion hydrogen-bonding scheme leads to a layered structure in this phase. The intrachain As...As separations of 4.2662 (3) and 4.3002 (4) $\AA$ in the $t$-butylammonium compound are significantly larger than those seen in (I).

\section{Experimental}

A $0.5 M$ aqueous creatine solution $(10 \mathrm{ml})$ was added to a $0.5 M$ aqueous $\mathrm{H}_{3} \mathrm{AsO}_{4}$ solution $(20 \mathrm{ml})$ to result in a clear solution. A mass of block-like crystals of (I) grew as the water evaporated over the course of a few days. The creatine transformed to creatinine under the low-pH conditions of the reaction. 


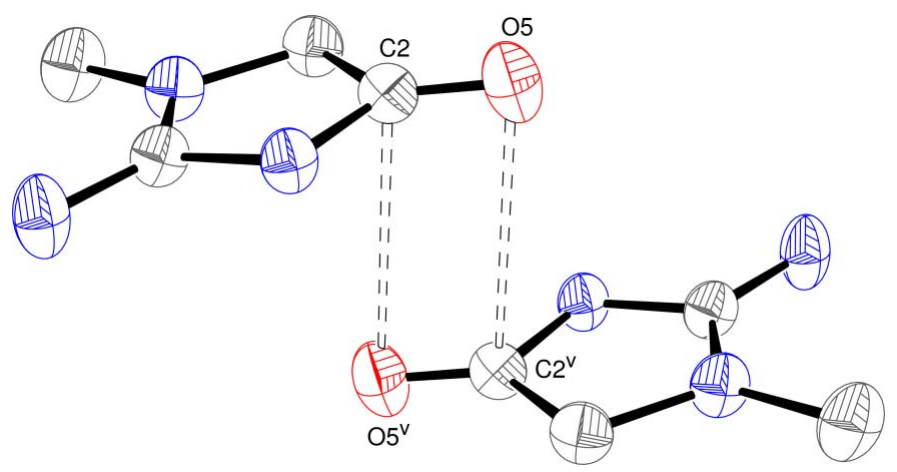

Figure 4

Detail of (I), showing the possible carbonyl-carbonyl interaction, with $\mathrm{H}$ atoms omitted for clarity. Hydrogen bonds are indicated by dashed lines. [Symmetry code: $(\mathrm{v})-x, 1-y,-z$.]

\section{Crystal data}

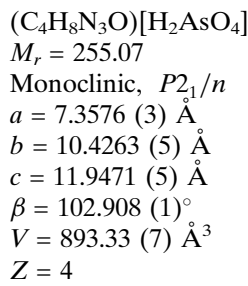

$D_{x}=1.897 \mathrm{Mg} \mathrm{m}^{-3}$

Mo $K \alpha$ radiation

Cell parameters from 5020 reflections

$\theta=2.6-32.2^{\circ}$

$\mu=3.80 \mathrm{~mm}^{-1}$

$T=293(2) \mathrm{K}$

Chunk, colourless

$0.49 \times 0.33 \times 0.24 \mathrm{~mm}$

\section{Data collection}

\section{Bruker SMART 1000 CCD} diffractometer

$\omega$ scans

Absorption correction: multi-scan (SADABS; Bruker, 1999)

$T_{\text {min }}=0.223, T_{\text {max }}=0.401$

11192 measured reflections

\section{Refinement}

Refinement on $F^{2}$

$R\left[F^{2}>2 \sigma\left(F^{2}\right)\right]=0.027$

$w R\left(F^{2}\right)=0.069$

$S=1.00$

3202 reflections

119 parameters

Table 1

Selected interatomic distances $(\AA)$.

\begin{tabular}{llll}
\hline As1-O1 & $1.6512(13)$ & $\mathrm{C} 1-\mathrm{N} 2$ & $1.305(2)$ \\
As1-O2 & $1.6563(12)$ & $\mathrm{C} 1-\mathrm{N} 3$ & $1.321(2)$ \\
As1-O4 & $1.7013(15)$ & $\mathrm{C} 1-\mathrm{N} 1$ & $1.374(2)$ \\
As1-O3 & $1.7134(13)$ & $\mathrm{C} 2-\mathrm{N} 1$ & $1.367(2)$ \\
\hline
\end{tabular}

3202 independent reflections 2403 reflections with $I>2 \sigma(I)$ $R_{\text {int }}=0.029$

$\theta_{\text {max }}=32.5^{\circ}$

$h=-11 \rightarrow 11$

$k=-15 \rightarrow 15$

$l=-17 \rightarrow 18$

H-atom parameters constrained $w=1 /\left[\sigma^{2}\left(F_{\mathrm{o}}{ }^{2}\right)+(0.0381 P)^{2}\right]$

where $P=\left(F_{\mathrm{o}}^{2}+2 F_{\mathrm{c}}^{2}\right) / 3$

$(\Delta / \sigma)_{\max }=0.002$

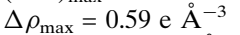

$\Delta \rho_{\min }=-0.52 \mathrm{e}^{-3}$
Table 2

Hydrogen-bond geometry $\left(\AA,^{\circ}\right)$.

\begin{tabular}{lllll}
\hline$D-\mathrm{H} \cdots A$ & $D-\mathrm{H}$ & $\mathrm{H} \cdots A$ & $D \cdots A$ & $D-\mathrm{H} \cdots A$ \\
\hline $\mathrm{O} 3-\mathrm{H} 1 \cdots \mathrm{O} 2^{\text {i }}$ & 0.85 & 1.77 & $2.618(2)$ & 177 \\
$\mathrm{O}^{2}-\mathrm{H} 2 \cdots \mathrm{O} 1^{\text {ii }}$ & 0.82 & 1.79 & $2.598(2)$ & 169 \\
$\mathrm{~N} 1-\mathrm{H} 3 \cdots \mathrm{O} 2$ & 0.86 & 1.89 & $2.703(2)$ & 158 \\
$\mathrm{~N} 2-\mathrm{H} 4 \cdots \mathrm{O} 5^{\text {iii }}$ & 0.86 & 2.16 & $2.983(2)$ & 161 \\
$\mathrm{~N} 2-\mathrm{H} 5 \cdots \mathrm{O} 1$ & 0.86 & 1.89 & $2.747(2)$ & 172 \\
$\mathrm{C} 3-\mathrm{H} 6 \cdots \mathrm{O} 1^{\text {iv }}$ & 0.97 & 2.46 & $3.334(2)$ & 149 \\
$\mathrm{C} 3-\mathrm{H} 7 \cdots \mathrm{O} 2^{\mathrm{v}}$ & 0.97 & 2.46 & $3.384(2)$ & 159
\end{tabular}

Symmetry codes: (i) $-x,-y+2,-z$; (ii) $-x+1,-y+2,-z$; (iii) $x+1, y, z$; (iv) $-x+\frac{1}{2}, y-\frac{1}{2},-z+\frac{1}{2} ;(\mathrm{v})-x,-y+1,-z$.

The hydroxy $\mathrm{H}$ atoms were found in difference maps and refined as riding on their carrier $\mathrm{O}$ atoms in their as-found relative positions. The $\mathrm{H}$ atoms bonded to $\mathrm{C}$ and $\mathrm{N}$ atoms were placed in idealized positions $(\mathrm{C}-\mathrm{H}=0.96-0.97 \AA$ and $\mathrm{N}-\mathrm{H}=0.86 \AA$ ) and refined as riding, allowing for free rotation of the $-\mathrm{CH}_{3}$ group. The constraint $U_{\text {iso }}(\mathrm{H})=1.2 U_{\text {eq }}$ (carrier) or $1.5 U_{\text {eq }}$ (methyl carrier) was applied.

Data collection: SMART (Bruker, 1999); cell refinement: SAINT (Bruker, 1999); data reduction: $S A I N T$; $\operatorname{program}(\mathrm{s})$ used to solve structure: SHELXS97 (Sheldrick, 1997); program(s) used to refine structure: SHELXL97 (Sheldrick, 1997); molecular graphics: ORTEP-3 (Farrugia, 1997); software used to prepare material for publication: SHELXL97.

HSW thanks the Carnegie Trust for the Universities of Scotland for an undergraduate vacation studentship.

\section{References}

Allen, F. H., Baalham, C. A., Lommerse, J. P. M. \& Raithby, P. R. (1998). Acta Cryst. B54, 320-329.

Bernstein, J., Davis, R. E., Shimoni, L. \& Chang, N.-L. (1995). Angew. Chem. Int. Ed. Engl. 34, 1555-1573.

Bondi, A. (1964). J. Phys. Chem. 68, 441-451.

Bruker (1999). SMART (Version 5.624), SAINT (Version 6.02A) and $S A D A B S$. Bruker AXS Inc., Madison, Wisconsin, USA.

Farrugia, L. J. (1997). J. Appl. Cryst. 30, 565.

Moghimi, A., Sharif, M. A. \& Aghabozorg, H. (2004). Acta Cryst. E60, o1790o1792.

Sheldrick, G. M. (1997). SHELXS97 and SHELXL97. University of Göttingen, Germany.

Spek, A. L. (2003). J. Appl. Cryst. 36, 7-13.

Todd, M. J. \& Harrison, W. T. A. (2005). Acta Cryst. E61, m1024-m1026.

Wilkinson, H. S. \& Harrison, W. T. A. (2004). Acta Cryst. E60, m1359m1361. 


\section{supporting information}

Acta Cryst. (2005). E61, m1228-m1230［https://doi.org/10.1107/S1600536805016144]

\section{Creatininium dihydrogenarsenate}

\section{Hazel S. Wilkinson and William T. A. Harrison}

Creatininium dihydrogenarsenate

Crystal data

$\left(\mathrm{C}_{4} \mathrm{H}_{8} \mathrm{~N}_{3} \mathrm{O}\right)\left[\mathrm{H}_{2} \mathrm{AsO}_{4}\right]$

$M_{r}=255.07$

Monoclinic, $P 2_{1} / n$

Hall symbol: -P 2 yn

$a=7.3576(3) \AA$

$b=10.4263(5) \AA$

$c=11.9471(5) \AA$

$\beta=102.908(1)^{\circ}$

$V=893.33(7) \AA^{3}$

$Z=4$

\section{Data collection}

Bruker SMART 1000 CCD diffractometer

Radiation source: fine-focus sealed tube Graphite monochromator $\omega$ scans

Absorption correction: multi-scan

(SADABS; Bruker, 1999)

$T_{\min }=0.223, T_{\max }=0.401$

Refinement

Refinement on $F^{2}$

Least-squares matrix: full

$R\left[F^{2}>2 \sigma\left(F^{2}\right)\right]=0.027$

$w R\left(F^{2}\right)=0.069$

$S=1.00$

3202 reflections

119 parameters

0 restraints

Primary atom site location: structure-invariant direct methods
$F(000)=512$

$D_{\mathrm{x}}=1.897 \mathrm{Mg} \mathrm{m}^{-3}$

Mo $K \alpha$ radiation, $\lambda=0.71073 \AA$

Cell parameters from 5020 reflections

$\theta=2.6-32.2^{\circ}$

$\mu=3.80 \mathrm{~mm}^{-1}$

$T=293 \mathrm{~K}$

Chunk, colourless

$0.49 \times 0.33 \times 0.24 \mathrm{~mm}$

11192 measured reflections 3202 independent reflections 2403 reflections with $I>2 \sigma(I)$

$R_{\text {int }}=0.029$

$\theta_{\max }=32.5^{\circ}, \theta_{\min }=2.6^{\circ}$

$h=-11 \rightarrow 11$

$k=-15 \rightarrow 15$

$l=-17 \rightarrow 18$

Secondary atom site location: difference Fourier map

Hydrogen site location: inferred from neighbouring sites

$\mathrm{H}$-atom parameters constrained

$w=1 /\left[\sigma^{2}\left(F_{\mathrm{o}}^{2}\right)+(0.0381 P)^{2}\right]$

where $P=\left(F_{\mathrm{o}}^{2}+2 F_{\mathrm{c}}{ }^{2}\right) / 3$

$(\Delta / \sigma)_{\max }=0.002$

$\Delta \rho_{\max }=0.59 \mathrm{e} \AA^{-3}$

$\Delta \rho_{\min }=-0.52$ e $\AA^{-3}$

Special details

Geometry. All e.s.d.'s (except the e.s.d. in the dihedral angle between two 1.s. planes) are estimated using the full covariance matrix. The cell e.s.d.'s are taken into account individually in the estimation of e.s.d.'s in distances, angles and torsion angles; correlations between e.s.d.'s in cell parameters are only used when they are defined by crystal symmetry. An approximate (isotropic) treatment of cell e.s.d.'s is used for estimating e.s.d.'s involving 1.s. planes. 
Refinement. Refinement of $F^{2}$ against ALL reflections. The weighted $R$-factor $w R$ and goodness of fit $S$ are based on $F^{2}$, conventional $R$-factors $R$ are based on $F$, with $F$ set to zero for negative $F^{2}$. The threshold expression of $F^{2}>\sigma\left(F^{2}\right)$ is used only for calculating $R$-factors(gt) etc. and is not relevant to the choice of reflections for refinement. $R$-factors based on $F^{2}$ are statistically about twice as large as those based on $F$, and $R$ - factors based on ALL data will be even larger.

Fractional atomic coordinates and isotropic or equivalent isotropic displacement parameters $\left(\AA^{2}\right)$

\begin{tabular}{lllll}
\hline & $x$ & $y$ & $z$ & $U_{\text {iso }} / U_{\text {eq }}$ \\
\hline As1 & $0.26213(2)$ & $0.928771(15)$ & $0.019997(18)$ & $0.03047(7)$ \\
O1 & $0.46360(18)$ & $0.87172(12)$ & $0.09239(13)$ & $0.0373(3)$ \\
O2 & $0.08787(18)$ & $0.82749(11)$ & $0.01759(13)$ & $0.0398(3)$ \\
O3 & $0.2283(2)$ & $1.06885(12)$ & $0.08753(15)$ & $0.0438(3)$ \\
H1 & 0.1274 & 1.1029 & 0.0514 & $0.053^{*}$ \\
O4 & $0.2634(2)$ & $0.96492(14)$ & $-0.11868(13)$ & $0.0444(3)$ \\
H2 & 0.3451 & 1.0179 & -0.1198 & $0.053^{*}$ \\
C1 & $0.3742(2)$ & $0.54747(15)$ & $0.13831(17)$ & $0.0311(4)$ \\
C2 & $0.0711(3)$ & $0.51202(17)$ & $0.13441(17)$ & $0.0338(4)$ \\
C3 & $0.1748(3)$ & $0.38752(17)$ & $0.16057(18)$ & $0.0355(4)$ \\
H6 & 0.1640 & 0.3537 & 0.2344 & $0.043^{*}$ \\
H7 & 0.1294 & 0.3240 & 0.1016 & $0.043^{*}$ \\
C4 & $0.5201(3)$ & $0.33417(18)$ & $0.1848(2)$ & $0.0446(5)$ \\
H8 & 0.4793 & 0.2528 & 0.1506 & $0.067^{*}$ \\
H9 & 0.5636 & 0.3239 & 0.2662 & $0.067^{*}$ \\
H10 & 0.6196 & 0.3664 & 0.1527 & $0.067^{*}$ \\
N1 & $0.2003(2)$ & $0.60209(13)$ & $0.12221(14)$ & $0.0316(3)$ \\
H3 & 0.1761 & 0.6817 & 0.1067 & $0.038^{*}$ \\
N2 & $0.5212(2)$ & $0.61337(15)$ & $0.12952(17)$ & $0.0430(4)$ \\
H4 & 0.6280 & 0.5763 & 0.1395 & $0.052^{*}$ \\
H5 & 0.5112 & 0.6939 & 0.1138 & $0.052^{*}$ \\
N3 & $0.3657(2)$ & $0.42406(13)$ & $0.16181(15)$ & $0.0345(3)$ \\
O5 & $-0.0931(2)$ & $0.53087(16)$ & $0.12300(15)$ & $0.0489(4)$ \\
& & & &
\end{tabular}

Atomic displacement parameters $\left(\AA^{2}\right)$

\begin{tabular}{lllllll}
\hline & $U^{11}$ & $U^{22}$ & $U^{33}$ & $U^{12}$ & $U^{13}$ & $U^{23}$ \\
\hline As1 & $0.02924(9)$ & $0.01985(8)$ & $0.03993(12)$ & $-0.00035(7)$ & $0.00263(7)$ & $0.00260(7)$ \\
O1 & $0.0290(6)$ & $0.0275(6)$ & $0.0517(9)$ & $0.0024(5)$ & $0.0011(6)$ & $0.0088(6)$ \\
O2 & $0.0305(6)$ & $0.0231(5)$ & $0.0629(10)$ & $-0.0022(5)$ & $0.0042(6)$ & $0.0075(6)$ \\
O3 & $0.0382(7)$ & $0.0294(6)$ & $0.0582(10)$ & $0.0038(6)$ & $-0.0012(7)$ & $-0.0102(6)$ \\
O4 & $0.0428(8)$ & $0.0446(7)$ & $0.0421(9)$ & $-0.0107(6)$ & $0.0013(7)$ & $0.0051(6)$ \\
C1 & $0.0325(8)$ & $0.0231(7)$ & $0.0364(10)$ & $0.0023(6)$ & $0.0051(7)$ & $0.0003(6)$ \\
C2 & $0.0349(9)$ & $0.0316(8)$ & $0.0351(10)$ & $-0.0010(7)$ & $0.0085(8)$ & $-0.0012(7)$ \\
C3 & $0.0384(10)$ & $0.0263(7)$ & $0.0418(11)$ & $-0.0038(7)$ & $0.0089(8)$ & $0.0015(7)$ \\
C4 & $0.0440(11)$ & $0.0260(8)$ & $0.0602(14)$ & $0.0085(8)$ & $0.0039(10)$ & $0.0030(8)$ \\
N1 & $0.0298(7)$ & $0.0230(6)$ & $0.0418(9)$ & $0.0031(5)$ & $0.0077(6)$ & $0.0028(6)$ \\
N2 & $0.0282(8)$ & $0.0269(7)$ & $0.0722(13)$ & $0.0026(6)$ & $0.0077(8)$ & $0.0076(8)$ \\
N3 & $0.0356(8)$ & $0.0216(6)$ & $0.0449(10)$ & $0.0017(6)$ & $0.0062(7)$ & $0.0021(6)$ \\
O5 & $0.0340(7)$ & $0.0476(8)$ & $0.0678(11)$ & $0.0002(7)$ & $0.0176(7)$ & $0.0017(8)$ \\
& & & & & & \\
\hline
\end{tabular}


Geometric parameters $\left(\AA,{ }^{\circ}\right)$

\begin{tabular}{|c|c|c|c|}
\hline Asl-O1 & $1.6512(13)$ & $\mathrm{C} 2-\mathrm{C} 3$ & $1.503(3)$ \\
\hline $\mathrm{As} 1-\mathrm{O} 2$ & $1.6563(12)$ & $\mathrm{C} 3-\mathrm{N} 3$ & $1.452(2)$ \\
\hline As $1-\mathrm{O} 4$ & $1.7013(15)$ & $\mathrm{C} 3-\mathrm{H} 6$ & 0.9700 \\
\hline $\mathrm{As} 1-\mathrm{O} 3$ & $1.7134(13)$ & $\mathrm{C} 3-\mathrm{H} 7$ & 0.9700 \\
\hline $\mathrm{O} 3-\mathrm{H} 1$ & 0.8488 & $\mathrm{C} 4-\mathrm{N} 3$ & $1.451(2)$ \\
\hline $\mathrm{O} 4-\mathrm{H} 2$ & 0.8185 & $\mathrm{C} 4-\mathrm{H} 8$ & 0.9600 \\
\hline $\mathrm{C} 1-\mathrm{N} 2$ & $1.305(2)$ & $\mathrm{C} 4-\mathrm{H} 9$ & 0.9600 \\
\hline $\mathrm{C} 1-\mathrm{N} 3$ & $1.321(2)$ & $\mathrm{C} 4-\mathrm{H} 10$ & 0.9600 \\
\hline $\mathrm{C} 1-\mathrm{N} 1$ & $1.374(2)$ & $\mathrm{N} 1-\mathrm{H} 3$ & 0.8600 \\
\hline $\mathrm{C} 2-\mathrm{O} 5$ & $1.202(2)$ & $\mathrm{N} 2-\mathrm{H} 4$ & 0.8600 \\
\hline $\mathrm{C} 2-\mathrm{N} 1$ & $1.367(2)$ & $\mathrm{N} 2-\mathrm{H} 5$ & 0.8600 \\
\hline $\mathrm{O} 1-\mathrm{As} 1-\mathrm{O} 2$ & $112.36(6)$ & $\mathrm{C} 2-\mathrm{C} 3-\mathrm{H} 7$ & 111.2 \\
\hline $\mathrm{O} 1-\mathrm{As} 1-\mathrm{O} 4$ & $112.96(7)$ & $\mathrm{H} 6-\mathrm{C} 3-\mathrm{H} 7$ & 109.1 \\
\hline $\mathrm{O} 2-\mathrm{As} 1-\mathrm{O} 4$ & $107.28(7)$ & $\mathrm{N} 3-\mathrm{C} 4-\mathrm{H} 8$ & 109.5 \\
\hline $\mathrm{O} 1-\mathrm{As} 1-\mathrm{O} 3$ & $105.56(7)$ & $\mathrm{N} 3-\mathrm{C} 4-\mathrm{H} 9$ & 109.5 \\
\hline $\mathrm{O} 2-\mathrm{As} 1-\mathrm{O} 3$ & $111.00(7)$ & $\mathrm{H} 8-\mathrm{C} 4-\mathrm{H} 9$ & 109.5 \\
\hline $\mathrm{O} 4-\mathrm{As} 1-\mathrm{O} 3$ & $107.62(8)$ & $\mathrm{N} 3-\mathrm{C} 4-\mathrm{H} 10$ & 109.5 \\
\hline $\mathrm{As} 1-\mathrm{O} 3-\mathrm{H} 1$ & 108.6 & $\mathrm{H} 8-\mathrm{C} 4-\mathrm{H} 10$ & 109.5 \\
\hline $\mathrm{As} 1-\mathrm{O} 4-\mathrm{H} 2$ & 109.2 & $\mathrm{H} 9-\mathrm{C} 4-\mathrm{H} 10$ & 109.5 \\
\hline $\mathrm{N} 2-\mathrm{C} 1-\mathrm{N} 3$ & $127.51(17)$ & $\mathrm{C} 2-\mathrm{N} 1-\mathrm{C} 1$ & $110.36(14)$ \\
\hline $\mathrm{N} 2-\mathrm{C} 1-\mathrm{N} 1$ & $122.20(16)$ & $\mathrm{C} 2-\mathrm{N} 1-\mathrm{H} 3$ & 124.8 \\
\hline $\mathrm{N} 3-\mathrm{C} 1-\mathrm{N} 1$ & $110.29(15)$ & $\mathrm{C} 1-\mathrm{N} 1-\mathrm{H} 3$ & 124.8 \\
\hline $\mathrm{O} 5-\mathrm{C} 2-\mathrm{N} 1$ & $125.52(18)$ & $\mathrm{C} 1-\mathrm{N} 2-\mathrm{H} 4$ & 120.0 \\
\hline $\mathrm{O} 5-\mathrm{C} 2-\mathrm{C} 3$ & $128.01(18)$ & $\mathrm{C} 1-\mathrm{N} 2-\mathrm{H} 5$ & 120.0 \\
\hline $\mathrm{N} 1-\mathrm{C} 2-\mathrm{C} 3$ & $106.45(15)$ & $\mathrm{H} 4-\mathrm{N} 2-\mathrm{H} 5$ & 120.0 \\
\hline $\mathrm{N} 3-\mathrm{C} 3-\mathrm{C} 2$ & $102.73(14)$ & $\mathrm{C} 1-\mathrm{N} 3-\mathrm{C} 4$ & $126.66(16)$ \\
\hline $\mathrm{N} 3-\mathrm{C} 3-\mathrm{H} 6$ & 111.2 & $\mathrm{C} 1-\mathrm{N} 3-\mathrm{C} 3$ & $110.16(15)$ \\
\hline $\mathrm{C} 2-\mathrm{C} 3-\mathrm{H} 6$ & 111.2 & $\mathrm{C} 4-\mathrm{N} 3-\mathrm{C} 3$ & $123.18(15)$ \\
\hline $\mathrm{N} 3-\mathrm{C} 3-\mathrm{H} 7$ & 111.2 & & \\
\hline $\mathrm{O} 5-\mathrm{C} 2-\mathrm{C} 3-\mathrm{N} 3$ & $179.0(2)$ & $\mathrm{N} 2-\mathrm{C} 1-\mathrm{N} 3-\mathrm{C} 4$ & $0.9(3)$ \\
\hline $\mathrm{N} 1-\mathrm{C} 2-\mathrm{C} 3-\mathrm{N} 3$ & $0.8(2)$ & $\mathrm{N} 1-\mathrm{C} 1-\mathrm{N} 3-\mathrm{C} 4$ & $-179.61(18)$ \\
\hline $\mathrm{O} 5-\mathrm{C} 2-\mathrm{N} 1-\mathrm{C} 1$ & $-178.5(2)$ & $\mathrm{N} 2-\mathrm{C} 1-\mathrm{N} 3-\mathrm{C} 3$ & $-178.4(2)$ \\
\hline $\mathrm{C} 3-\mathrm{C} 2-\mathrm{N} 1-\mathrm{C} 1$ & $-0.2(2)$ & $\mathrm{N} 1-\mathrm{C} 1-\mathrm{N} 3-\mathrm{C} 3$ & $1.0(2)$ \\
\hline $\mathrm{N} 2-\mathrm{C} 1-\mathrm{N} 1-\mathrm{C} 2$ & $179.00(19)$ & $\mathrm{C} 2-\mathrm{C} 3-\mathrm{N} 3-\mathrm{C} 1$ & $-1.1(2)$ \\
\hline $\mathrm{N} 3-\mathrm{C} 1-\mathrm{N} 1-\mathrm{C} 2$ & $-0.5(2)$ & $\mathrm{C} 2-\mathrm{C} 3-\mathrm{N} 3-\mathrm{C} 4$ & $179.53(18)$ \\
\hline
\end{tabular}

Hydrogen-bond geometry $\left(\AA,{ }^{\circ}\right)$

\begin{tabular}{lllll}
\hline$D-\mathrm{H} \cdots A$ & $D-\mathrm{H}$ & $\mathrm{H} \cdots A$ & $D \cdots A$ & $D-\mathrm{H} \cdots A$ \\
\hline $\mathrm{O} 3-\mathrm{H} 1 \cdots \mathrm{O} 2^{\mathrm{i}}$ & 0.85 & 1.77 & $2.618(2)$ & 177 \\
$\mathrm{O} 4-\mathrm{H} 2 \cdots \mathrm{O} 1^{\mathrm{ii}}$ & 0.82 & 1.79 & $2.598(2)$ & 169 \\
$\mathrm{~N} 1-\mathrm{H} 3 \cdots \mathrm{O} 2$ & 0.86 & 1.89 & $2.703(2)$ & 158 \\
$\mathrm{~N} 2-\mathrm{H} 4 \cdots \mathrm{O} 5^{\mathrm{iii}}$ & 0.86 & 2.16 & $2.983(2)$ & 161 \\
$\mathrm{~N} 2-\mathrm{H} 5 \cdots \mathrm{O} 1$ & 0.86 & 1.89 & $2.747(2)$ & 172
\end{tabular}


supporting information

$\begin{array}{llllr}\mathrm{C} 3-\mathrm{H} 6 \cdots \mathrm{O} 1^{\text {iv }} & 0.97 & 2.46 & 3.334(2) & 149 \\ \mathrm{C} 3-\mathrm{H} 7 \cdots 2^{\mathrm{v}} & 0.97 & 2.46 & 3.384(2) & 159\end{array}$

Symmetry codes: (i) $-x,-y+2,-z$; (ii) $-x+1,-y+2,-z$; (iii) $x+1, y, z$; (iv) $-x+1 / 2, y-1 / 2,-z+1 / 2$; (v) $-x,-y+1,-z$. 